\title{
The Cloud Computing Load Forecasting Algorithm Based on Kalman Filter and ANFIS
}

\author{
Jian Sun ${ }^{1, a}$, Yi Zhuang ${ }^{1, b}$ \\ ${ }^{1}$ College of Computer Science and Technology, Nanjing University of Aeronautics and Astronautics, \\ Nanjing, 210016, China \\ a470432534@qq.com, by16@nuaa.edu.com,
}

Keywords: cloud computing, load forecasting, Kalman Filter, ANFIS

\begin{abstract}
The load forecasting in the cloud computing is one of the most important technologies to ensure the maximize utilization of the system resource. Under the premise that the load is known in the next stage, the cloud computing center can assign the physical machines in advance, thereby reducing the waiting time of the task, and can also reduce the cloud computing center energy consumption. This paper proposed a load forecasting algorithm based on the Kalman filter and adaptive neuro-fuzzy inference system (ANFIS), obtained more accurate load sequence by the kalman filter eliminate observation error, used ANFIS to forecast the load sequence. The predicted results were compared with the original ANFIS algorithm, Autoregressive Integrated Moving Average (ARIMA) algorithm. The K-ANFIS algorithm had improved the prediction accuracy significantly compared with the other two algorithms.
\end{abstract}

\section{Introduction}

The concept of infrastructure as a service, software as a service and platform as a service enables the rapid development of the commercial cloud computing center, at the same time, the energy consumption is also becoming increasingly large. Large-scale cloud computing center needs to consume hundreds of millions of electricity to maintain the operation of the physical machines, also needs quite a lot of power to carry out physical machine cooling and other peripheral services. It has become the key to improve the utility in cloud computing that improve the utilization of the resources. Cloud computing load forecasting technology can analysis the previous load and according to these data predict the next stage load, the forecast data can be used to adjust the physical machines so as to reduce the waiting time of the task and achieve the purpose of saving energy. Therefore, load forecasting technology as a key technology to save energy consumption and improve the utility of cloud computing center, has been widespread concerned and researched by scholars in recent years along with resource allocation and virtual machine migration.

\section{Related Research}

Cloud computing load forecasting is relatively less in the domestic research. Zhang Lei et al. proposed a multi-step optimization of $\operatorname{GM}(1,1)$ model [1], which can forecast the load in cloud computing in short-term. The model was verified by public available data of NASA and Clarknet, and the results were compared with Autoregressive Moving Average (ARMA) and Back Propagation (BP) algorithm, indicating that the prediction accuracy has been improved. However, the model is validated in short-term, the performance is unknown when the forecast time grow. Xu Dayu et al. studied the method of resource demand prediction and allocation optimize in cloud computing, proposed short-term load forecasting method of dynamic cloud resources based on feature extraction and classification and long-term load forecasting method of combination forecast algorithm based on generalized fuzzy set theory [2]. Respectively, using Google Cloud Computing Center dataset and the NASA data sets to verify the prediction algorithm, and the predicted results were compared with some other algorithms. The results show that these two algorithms in short-term prediction and long-term prediction is indeed better than some of the existing traditional algorithm, but the two 
models of the algorithms is relatively more load, not suitable for in the cloud computing environment in real time on task load forecasting.

Study relatively early abroad on cloud computing load forecasting, and research results are also relatively rich. M López et al. proposed a standard for short-term load forecasting [3], which includes not only the selection of prediction algorithm, but also the establishment of prediction model for data selection, forecasting data preprocessing and so on. Prevost $\mathrm{J} \mathrm{J}$ et al. used the autoregressive method linear regression prediction and neural network prediction [4], and the prediction accuracy and prediction length was tested. Sheng D et al. applied the Bayesian algorithm to load forecasting [5], by selecting the characteristic of nine dimensions, Bayesian algorithm can well predict the next phase of the task load average value. Rasheduzzaman $\mathrm{M}$ et al. studied different load forecasting algorithms and compared the accuracy of the prediction [6], the results show that ANFIS algorithm has lower error rate.

This paper proposes a forecasting algorithm base on preprocessing, for the volatility characteristic of the load in cloud computing, the first use Kalman filtering its filtering process, resulting in more accurately reflect the characteristics of cloud computing load curve, then use ANFIS to forecast the load for the next phase of the load. This paper compares the proposed method with several other prediction methods, the results show that the proposed method improves the accuracy of the prediction.

\section{K-ANFIS algorithm}

Kalman filtering.In 1960, American scientist R.E.Kalman established Kalman filtering algorithm for discrete stochastic systems based on Wiener filtering, also can processing for linear continuous time systems [7]. General discrete systems can be expressed as formula (1) [8].

$$
\begin{aligned}
& X(k+1)=\Phi(k+1, k) X(k)+\Gamma(k+1, k) w(k) \\
& Z(k+1)=H(k+1) X(k+1)+v(k+1)
\end{aligned}
$$

In formula (1), $X(k)$ is the n-dimensional state vector, $Z(k)$ is the m-dimensional observation vector, $w(k)$ is the p-dimensional state vector, $v(k+1)$ is the m-dimensional noise vector measurement. $\Phi(k+1, k)$ is the state transition matrix from the time $k$ to time $k+1, \Gamma(k+1, k)$ is the incentive transfer matrix from time $k$ to time $k+1, H(k+1)$ predictive output matrix for $k+1$ moments.

K-ANFIS algorithm. The ANFIS model was developed by Artificial Neural Network (ANN). In 1985, Sugeno proposed a kind of T-S fuzzy model, the fuzzy model is nonlinear model, easy to express dynamic characteristics of load system [9]. On this basis, the ANFIS system, which is developed from one order Sugeno fuzzy model, is a kind of neural network model based on fuzzy reasoning, which has the advantages of fuzzy inference system and neural network system. The system is divided into three parts, one is the learning ability of the fuzzy rule set IF-THEN, two is the membership function of the definition of the data set, three is the corresponding reasoning system. The two most commonly used learning algorithms in ANFIS are the BP algorithm and the least square estimation algorithm. The typical ANFIS structure is shown in Figure (1) [9].

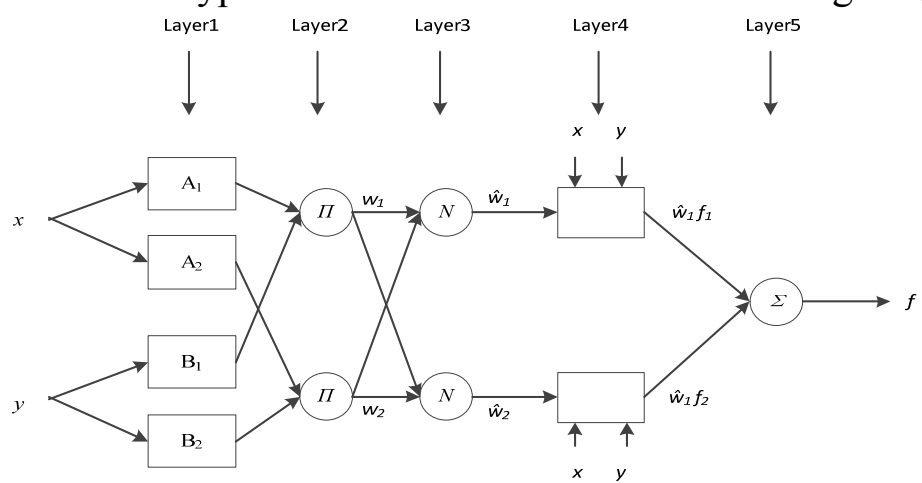

Figure 1 typical structure of ANFIS 
The block in the diagram represents a node with learning parameters. A two rule ANFIS Sugeno has the following form of rule [10].

if $x$ is $A_{1}$ AND $y$ is $B_{1}$ then $f_{1}=p_{1} x+q_{1} y+r_{1}$

if $x$ is $A_{2}$ AND $y$ is $B_{2}$ then $f_{2}=p_{2} x+q_{2} y+r_{2}$

In the structure of ANFIS, the first layer is responsible for the ambiguity of the input signal. Node $i$ has an output function, $x$ is the input of node $i$ and $A_{i}$ is the fuzzy set. $O_{i}^{1}$ is the member function value of $A_{i}$, which indicates that $x$ belongs to the degree. Typically chosen $\mu_{A_{i}}(x)$ to bell function and has a maximum 1, minimum $0 . a_{i}, b_{i}$ and $c_{i}$ are the premise parameters. The shape of the membership function changes with the change of these parameters. As shown in formula (3) [10].

$O_{i}^{1}=\mu_{A_{i}}(x)=\frac{1}{1+\left[\left(\frac{x-c_{i}}{a_{i}}\right)^{2}\right]^{b_{i}}}$

The second layer is responsible for the multiplication of the input signal, the output of each node represents the credibility of a rule. As shown in formula (4) [10].

$O_{i}^{2}=w_{i}=\mu_{A_{i}}(x) \times \mu_{B_{i}}(y) \quad i=1,2$

The third layer of the i-node calculation i rule normalized credibility. Shown in formula (5) [10].

$O_{i}^{3}=\overline{w_{i}}=\frac{w_{i}}{w_{1}+w_{2}} \quad i=1,2$

The i-th node of the fourth layer has the following output as formula (6) [10], wherein $\overline{w_{i}}$ is the output of the third layer, $p_{i}, q_{i}$ and $r_{i}$ are the parameters for the conclusion.

$O_{i}^{4}=\overline{w_{i}} f_{i}=\overline{w_{i}}\left(p_{i} x+q_{i} y+r_{i}\right)$

(6)

The fifth layer calculates the total output. As shown in formula (7) [10].

$O_{i}^{5}=\sum \overline{w_{i}} f_{i}=\frac{\sum_{i} \bar{w}_{i} f_{i}}{\sum_{i} w_{i}}$

\section{Experiment and Analysis}

To test the predictive performance of the proposed K-ANFIS algorithm in this paper, select Google cluster trace to do the experiment, this data set recorded of Google cloud computing center of the task load situation in nearly a month's time. Set the number of iterations to 100 , the number of membership function 5, predict the load sequence after Kalman filter with ANFIS algorithm. At the same time, using the same set of ANFIS algorithm, ARIMA algorithm to predict the untreated load sequence, and the results are compared with the algorithm proposed in this paper. The results are shown in figure 2(a). 


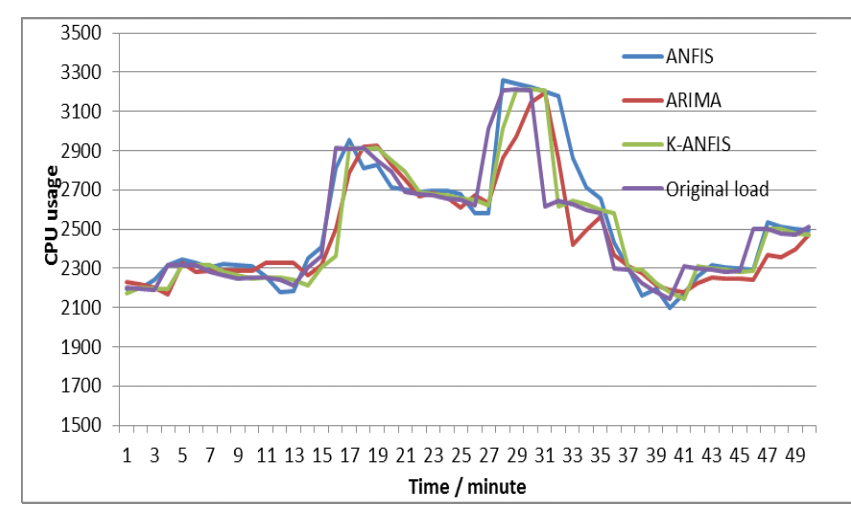

(a) the results of different prediction algorithms

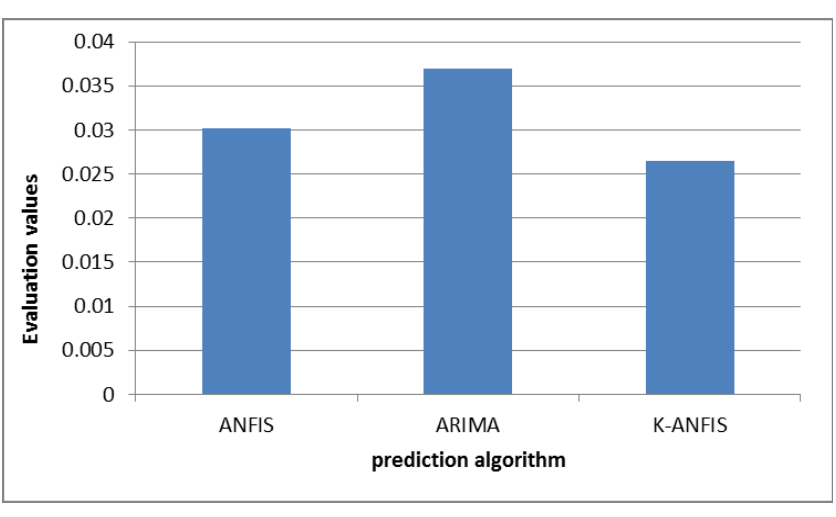

(b) Evaluation values of different prediction algorithm

Figure 2 Comparison of different prediction algorithms

The Mean Absolute Error (MAE) is chosen as the evaluation index, and the calculation method is shown in formula (8) [6].

$$
M A E=\frac{1}{n} \sum_{i=1}^{n}\left|\frac{y_{i}-f_{i}}{y_{i}}\right|
$$

The evaluation values of the three different algorithms in the MAE evaluation index are shown in figure 2(b).

According to the above results, the K-ANFIS algorithm proposed in this paper is better than the original ANFIS algorithm and ARIMA algorithm, and has better prediction accuracy in the cloud computing load forecasting.

\section{Conclusion}

In this paper, a cloud computing load forecasting algorithm based on Kalman filter and ANFIS is proposed. The cloud computing load is filtered by Kalman filter, and then the ANFIS algorithm is used to predict the load. The Google cluster trace was used to verify the performance, and compared with the other two algorithms, the results show that the proposed K-ANFIS algorithm has a better prediction accuracy.

\section{References}

[1] ZHANG Lei, XU Dayu. Multi-step optimized GM(1,1)model-based short term resource load prediction in cloud computing. Computer Engineering and Applications, 2014, 50 (10) : 65-71.

[2] Xu Dayu. On-demanded Resource Prediction and Optimal Resource Allocation Method Research in Cloud Computing Environment[D]. Hefei University of Technology,2014.

[3] López M, Valero S, Senabre C, et al. Standardization of short-term load forecasting models[C]// European Energy Market (EEM), 2012 9th International Conference on the. IEEE, 2012:1-7.

[4] Prevost J J, Nagothu K M, Kelley B, et al. Prediction of cloud data center networks loads using stochastic and neural models[C]// System of Systems Engineering (SoSE), 2011 6th International Conference on. IEEE, 2011:276 - 281.

[5] Sheng D, Kondo D, Cirne W. Host load prediction in a Google compute cloud with a Bayesian model[C]// SC Conference. IEEE, 2012:1-11.

[6] Rasheduzzaman M, Islam M A, Islam T, et al. Study of different forecasting models on Google cluster trace[C]// Computer and Information Technology (ICCIT), 2013 16th International Conference on. IEEE, 2014:414-419. 
[7] Dan Yang, A Research on Design and Application of Kalman Filter[D]. Xiangtan University, 2014. [5] Sheng D, Kondo D, Cirne W. Host load prediction in a Google compute cloud with a Bayesian model[C]// SC Conference. IEEE, 2012:1-11.

[8] Difu P, Hui L, Yanfei L. A wind speed forecasting optimization model for wind farms based on time series analysis and Kalman filter algorithm[J]. POWER SYSTEM TECHNOLOGY-BEIJING-, 2008, 32(7): 82.

[9] ZHANG H, YU Y, WANG Q. Modeling and Simulation Applying Adaptive Neural-Fuzzy Inference System (ANFIS)[J][J]. Computer Simulation, 2002, 4: 012.

$[10] \mathrm{Du}$ T. Forecasting Method of the Slope Displacement Based on the ANFIS Model[J]. JOURNAL-CHONGQING JIANZHU UNIVERSITY, 2005, 27(4): 55. 